Japan. J. Math.

Vol. 30, No. 2, 2004

\title{
Volterra difference equations on a Banach space and abstract differential equations with piecewise continuous delays
}

\author{
By Tetsuo Furumochi, Satoru Murakami* and Yutaka Nagabuchi \\ (Received July 2, 2002) \\ (Revised October 7, 2003) \\ (Communicated by Journal of Mathematical Sciences, the University of Tokyo)
}

\begin{abstract}
We obtain a condition on the spectrum of the characteristic operator for the zero solution of Volterra difference equations on a Banach space to be exponentially asymptotically stable. Moreover, we apply some results obtained here to derive stability properties for some differential equations with piecewise continuous delays.
\end{abstract}

\section{Introduction}

As shown in $[5,6,7,10]$ and other literatures, the study of some differential equations with piecewise continuous delays is reduced to the one of difference equations. Indeed, some Volterra difference equations on a Banach space are derived from some abstract differential equations with piecewise continuous delays as seen in Section 3.

In this paper we will first treat the following Volterra difference equation on a Banach space $X$

$$
x(n+1)=\sum_{j=-\infty}^{n} Q(n-j) x(j), \quad n \in \mathbf{Z}^{+}:=\{0,1,2, \ldots\},
$$

where $Q(n), \quad n \in \mathbf{Z}^{+}$, are bounded linear operators on $X$ such that $\sum_{n=0}^{\infty}\|Q(n)\| e^{\gamma n}<\infty$ for some positive constant $\gamma$, and study the stability property of the zero solution of Eq. (1). When $X$ is finite dimensional, there are several results on the stabilities in Eq. (1); see e.g. [3, 4] and the references therein. In Section 2, we will study the exponential asymptotic stability of the zero solution of

2000 Mathematics Subject Classification. Primary 39A10, 39A11; Secondary 34K20, 35B35.

Key words and Phrases. Volterra difference equations, stabilities, piecewise continuous delays, solution operator, $Z$ transform.

*The second author is partly supported by the Grant-in-Aid for Scientific Research (C), No. 16540177, of the Japanese Ministry of Education, Culture, Sports, Sciences and Technology. 
Eq. (1) under some condition on the spectrum of the corresponding characteristic operator, and extend some results in the case of finite dimensional $X$ to the case of inifinite dimensional $X$ (Theorems 1 and 2).

In fact, it may be rather difficult to check the condition on the spectrum of the characteristic operator. For difference equations which are induced by some special differential equations with piecewise continuous delays, however, one can overcome the difficulties. Indeed, in Theorem 3 we will characterize the spectrum of the characteristic operator by using the Gelfand transform in the theory of commutative Banach algebras.

Finally, we will treat a scalar partial differential equation with piecewise continuous delays which describes the population dynamics in a single species taking diffusion effects into consideration, and obtain a result on stability properties of a positive constant solution of the equation by reducing the equation to a difference equation on a Banach space and applying Theorem 3 to the reduced difference equation (Theorem 4).

\section{Stabilities in Volterra difference equations on a Banach space}

Let us denote by $\mathbf{Z}, \mathbf{Z}^{+}$and $\mathbf{Z}^{-}$the set of all integers, the set of all nonnegative integers and the set of all nonpositive integers, respectively. For a Banach space $X$ (with norm $|\cdot|$ ) over the field $\mathbf{C}$ of all the complex numbers, we denote by $\mathcal{L}(X)$ the space of all bounded linear operators on $X$, and define the norm of any $Q$ belonging to $\mathcal{L}(X)$ by

$$
\|Q\|=\sup \{|Q x|: x \in X,|x|=1\} .
$$

We now consider the Volterra difference equation on $X$

$$
x(n+1)=\sum_{j=-\infty}^{n} Q(n-j) x(j), \quad n \in \mathbf{Z}^{+},
$$

where $Q(n) \in \mathcal{L}(X)$. Throughout the paper, we assume the following condition on Eq. (2):

$$
\exists \gamma>0, l \geq 1: \quad \sum_{n=0}^{\infty}\|Q(n)\| e^{\gamma n} \leq l<\infty .
$$

For Eq. (2) we consider the operators $R(n), n \in \mathbf{Z}^{+}$, in $\mathcal{L}(X)$ defined by the relation

$$
R(n+1)=\sum_{j=0}^{n} Q(n-j) R(j), \quad n=0,1,2, \ldots
$$

and $R(0)=I$ (the identity operator on $X) . R(n)$ is called the fundamental solution 
of Eq. (2). By virtue of the inequality

$$
\begin{aligned}
\|R(n+1)\| & =\|Q(n) R(0)+Q(n-1) R(1)+\cdots+Q(0) R(n)\| \\
& \leq l \max _{0 \leq s \leq n}\|R(s)\|,
\end{aligned}
$$

we get the following estimate on $\|R(n)\|$ :

$$
\|R(n)\| \leq l^{n}, \quad n \in \mathbf{Z}^{+} .
$$

Therefore, the $Z$-transform

$$
\tilde{R}(z)=\sum_{n=0}^{\infty} R(n) z^{-n}
$$

of $R(n), n \in \mathbf{Z}^{+}$, exists in the domain $|z|>l$ of the complex plane, and it is analytic in the domain.

We say that $R(n)$ decays exponentially, if there exist positive constants $M$ and $\epsilon$ such that

$$
\|R(n)\| \leq M e^{-\epsilon n}, \quad n \in \mathbf{Z}^{+} .
$$

In the following we will give a condition for $R(n)$ to decay exponentially, in connection with the invertibility of $z I-\tilde{Q}(z)$ which is called the characteristic operator of Eq. (2).

Theorem 1. Suppose that the following condition holds true:

$$
(z I-\tilde{Q}(z))^{-1} \in \mathcal{L}(X) \quad(\forall|z| \geq 1) .
$$

Then the fundamental solution $R(n)$ of $E q$. (2) decays exponentially.

Conversely, in case where $Q(j), j=0,1,2, \ldots$ are compact operators, if $R(n)$ decays exponentially, then the condition (4) holds true.

Proof. We will first establish the first part of the theorem. Let us consider the $Z$ transform of the both sides of (3) to get

$$
z \tilde{R}(z)-z I=\tilde{Q}(z) \tilde{R}(z) \quad(|z|>l) .
$$

The operators $z I-\tilde{Q}(z),|z| \geq 1$, are invertible by virtue of the condition (4), and hence we get the following relation

$$
z(z I-\tilde{Q}(z))^{-1}=\tilde{R}(z) \quad(|z|>l) .
$$

Notice that $z I-\tilde{Q}(z)$ is invertible for each $z$ with $|z|=1$, and consequently so is for any point belonging to some open neighborhood of $z$ with $|z|=1$. Since the set $|z|=1$ is compact in the complex plane, there is a positive constant $\delta_{1} \in\left(0,1-e^{-\gamma}\right)$ 
such that the operator $z I-\tilde{Q}(z)$ is invertible in $\mathcal{L}(X)$ for any $z$ such that $|z| \geq 1-\delta_{1}$. Then the function $G(z):=z(z I-\tilde{Q}(z))^{-1}$ is analytic on the domain $|z|>1-\delta_{1}$, and it can be expanded into the Laurent series

$$
G(z)=\sum_{m \in \mathbf{Z}} b(m) z^{m} \quad\left(\forall|z|>1-\delta_{1}\right)
$$

where

$$
b(m)=\frac{1}{2 \pi i} \int_{|z|=L} \frac{G(z)}{z^{m+1}} d z \quad\left(L>1-\delta_{1}\right) .
$$

Let $|z|=L>l$. Since $l \geq 1$, we get $|z| e^{\gamma}>l e^{\gamma}>\left(1-\delta_{1}\right) e^{\gamma}>1$, and

$$
\begin{aligned}
\|\tilde{Q}(z)\|=\left\|\sum_{n=0}^{\infty} Q(n) z^{-n}\right\| & \leq \sum_{n=0}^{\infty}\|Q(n)\||z|^{-n} \\
& \leq \sum_{n=0}^{\infty}\|Q(n)\| e^{\gamma n}=l<|z|
\end{aligned}
$$

which yields that

$$
\|G(z)\|=\left\|\left(I-\frac{\tilde{Q}(z)}{z}\right)^{-1}\right\|=\left\|\sum_{n=0}^{\infty}\left(\frac{\tilde{Q}(z)}{z}\right)^{n}\right\| \leq \sum_{n=0}^{\infty}\left(\frac{l}{L}\right)^{n}=\frac{L}{L-l} .
$$

Therefore, for $n=1,2, \ldots$ we have

$$
\begin{aligned}
\|b(n)\|=\left\|\frac{1}{2 \pi i} \int_{|z|=L} \frac{G(z)}{z^{n+1}} d z\right\| & \leq\left[\sup _{|z|=L}\|G(z)\|\right] L^{-n} \\
& \leq \frac{L}{L^{n}(L-l)} \rightarrow 0 \quad(L \rightarrow \infty) .
\end{aligned}
$$

Then $b(n)=0(n=1,2, \ldots)$, and hence

$$
G(z)=\sum_{n=0}^{\infty} b(-n) z^{-n} \quad\left(\forall|z|>1-\delta_{1}\right) .
$$

In particular, the series $\sum_{n=0}^{\infty} b(-n)\left(1-\frac{\delta_{1}}{2}\right)^{-n}$ is convergent. Consequently, it follows that $\sup _{n \in \mathbf{Z}^{+}}\left\|b(-n)\left(1-\frac{\delta_{1}}{2}\right)^{-n}\right\|=: M<\infty$, and

$$
\|b(-n)\| \leq M\left(1-\frac{\delta_{1}}{2}\right)^{n}, \quad n \in \mathbf{Z}^{+} .
$$


Let us take an $L$ with $L>l$. Since

$$
\sum_{n=0}^{\infty} R(n) z^{-n}=\tilde{R}(z)=G(z)=\sum_{n=0}^{\infty} b(-n) z^{-n} \quad(\forall|z|=L)
$$

by (5), it follows that

$$
R(n)=\frac{1}{2 \pi i} \int_{|z|=L} \tilde{R}(z) z^{n-1} d z=\frac{1}{2 \pi i} \int_{|z|=L} G(z) z^{n-1} d z=b(-n), \quad n \in \mathbf{Z}^{+} .
$$

Put $\epsilon=-\log \left(1-\frac{\delta_{1}}{2}\right)$. Then $\epsilon>0$ and

$$
\|R(n)\|=\|b(-n)\| \leq M\left(1-\frac{\delta_{1}}{2}\right)^{n}=M e^{-\epsilon n}, \quad n \in \mathbf{Z}^{+}
$$

by (6), which shows that $R(n)$ decays exponentially.

Next we will establish the second part of the theorem. Assume that $\|R(n)\| \leq$ $M e^{-\epsilon n}, n \in \mathbf{Z}^{+}$, for some positive constants $M$ and $\epsilon$. Without loss of generality, one can assume that $0<\epsilon<\gamma$. Now suppose that the condition (4) does not hold true, that is, the operator $z_{0} I-\tilde{Q}\left(z_{0}\right)$ is not invertible for some $z_{0}$ with $\left|z_{0}\right| \geq 1$. Observe that $\sum_{j=0}^{\infty} Q(j) z_{0}^{-j}$ is a compact operator, because it is the limit of compact operators $\sum_{j=0}^{n} Q(j) z_{0}^{-j}$ in the operator norm as $n \rightarrow \infty$. Then the Riesz-Schauder theorem on compact operators (e.g., [9, Theorem 4.25]) implies that $z_{0}$ belongs to the point spectrum of $\tilde{Q}\left(z_{0}\right)$, and hence there exists a nonzero element $x_{0} \in X$ such that $\left(z_{0} I-\tilde{Q}\left(z_{0}\right)\right) x_{0}=0$, or $z_{0} x_{0}=\sum_{j=0}^{\infty} Q(j) z_{0}^{-j} x_{0}$.

Let us consider a function $x: \mathbf{Z} \rightarrow X$ defined by $x(n)=z_{0}^{n} x_{0}, n \in \mathbf{Z}$. For any $n \in \mathbf{Z}^{+}$, we get

$$
\begin{aligned}
x(n+1)=z_{0}^{n+1} x_{0}=z_{0}^{n}\left(\sum_{j=0}^{\infty} Q(j) z_{0}^{-j} x_{0}\right) & =\sum_{j=0}^{\infty} Q(j) x(n-j) \\
& =\sum_{j=0}^{n} Q(n-j) x(j)+h(n),
\end{aligned}
$$

where $h(n):=\sum_{j=-\infty}^{-1} Q(n-j) z_{0}^{j} x_{0}$. Put

$$
v(n)=R(n) x_{0}+\sum_{k=0}^{n-1} R(n-k-1) h(k), \quad n \in \mathbf{Z}^{+} .
$$

Here and hereafter, we employ the convention $\sum_{0}^{-1}=0$. It is clear that $v(0)=x_{0}$, 
and moreover, for $n \in \mathbf{Z}^{+}$we obtain

$$
\begin{aligned}
\sum_{j=0}^{n} Q(n-j) v(j) & =\sum_{j=0}^{n} Q(n-j)\left(R(j) x_{0}+\sum_{k=0}^{j-1} R(j-k-1) h(k)\right) \\
& =\sum_{j=0}^{n} Q(n-j) R(j) x_{0}+\sum_{k=0}^{n-1}\left(\sum_{j=k+1}^{n} Q(n-j) R(j-k-1)\right) h(k) \\
& =R(n+1) x_{0}+\sum_{k=0}^{n-1}\left(\sum_{\tau=0}^{n-k-1} Q(n-k-1-\tau) R(\tau)\right) h(k) \\
& =R(n+1) x_{0}+\sum_{k=0}^{n-1} R(n-k) h(k)
\end{aligned}
$$

and hence

$$
\begin{aligned}
v(n+1) & =R(n+1) x_{0}+\sum_{k=0}^{n} R(n-k) h(k) \\
& =\sum_{j=0}^{n} Q(n-j) v(j)+h(n), \quad n \in \mathbf{Z}^{+} .
\end{aligned}
$$

Then it follows from the uniqueness of the initial value problems that $x(n) \equiv v(n)$ or

$$
\begin{aligned}
x(n) & =R(n) x_{0}+\sum_{k=0}^{n-1} R(n-k-1) h(k) \\
& =R(n) x_{0}+\sum_{k=0}^{n-1} R(n-k-1)\left(\sum_{j=-\infty}^{-1} Q(k-j) z_{0}^{j} x_{0}\right), \quad n \in \mathbf{Z}^{+} .
\end{aligned}
$$

Since $|x(n)| \geq\left|x_{0}\right|(\forall n \geq 0)$ and since $\left|z_{0}\right|^{j} \leq 1(\forall j \leq 0)$, it follows that

$$
0<\left|x_{0}\right| \leq|x(n)| \leq\left\{\|R(n)\|+\sum_{k=0}^{n-1}\|R(n-k-1)\|\left(\sum_{j=-\infty}^{-1}\|Q(k-j)\|\right)\right\}\left|x_{0}\right|,
$$

or

$$
\begin{aligned}
1 & \leq\left\{\|R(n)\|+\sum_{k=0}^{n-1}\|R(n-k-1)\|\left(\sum_{j=-\infty}^{-1}\|Q(k-j)\|\right)\right\} \\
& \leq M e^{-\epsilon n}\left\{1+e^{\epsilon} \sum_{k=0}^{n-1} e^{(\epsilon-\gamma) k}\left(\sum_{j=-\infty}^{-1}\|Q(k-j)\| e^{\gamma(k-j)}\right)\right\}
\end{aligned}
$$




$$
\leq M e^{-\epsilon n}\left(1+\frac{l e^{\epsilon}}{1-e^{\epsilon-\gamma}}\right), \quad n \in \mathbf{Z}^{+}
$$

Letting $n \rightarrow \infty$ in the above inequality, we get $1 \leq 0$, a contradiction. Thus the condition (4) must hold true. This completes the proof of the theorem.

Let us consider the Banach space $\mathcal{B}^{\gamma}$ defined by

$$
\mathcal{B}^{\gamma}=\left\{\phi: \mathbf{Z}^{-} \rightarrow X\left|\sup _{\theta \in \mathbf{Z}^{-}}\right| \phi(\theta) \mid e^{\gamma \theta}<\infty\right\}
$$

equipped with the norm

$$
\|\phi\|=\sup _{\theta \in \mathbf{Z}^{-}}|\phi(\theta)| e^{\gamma \theta}, \quad \phi \in \mathcal{B}^{\gamma} .
$$

For any $\phi \in \mathcal{B}^{\gamma}$, it follows that

$$
\begin{aligned}
\sum_{j=-\infty}^{-1}\|Q(n-j) \phi(j)\| & \leq\left(\sum_{j=-\infty}^{-1}\|Q(n-j)\| e^{\gamma(n-j)}\right)\|\phi\| e^{-\gamma n} \\
& \leq l\|\phi\| e^{-\gamma n}, \quad n \in \mathbf{Z}^{+}
\end{aligned}
$$

Hence, if we set

$$
p(n):=\sum_{j=-\infty}^{-1} Q(n-j) \phi(j), \quad n \in \mathbf{Z}^{+},
$$

$p(n)$ is well-defined, and there exists a unique solution $x$ of Eq. (2) on $\mathbf{Z}^{+}$such that $x(\theta) \equiv \phi(\theta), \theta \in \mathbf{Z}^{-}$. We denote such a solution by $x(\cdot ; \phi)$. Then $x(\cdot ; \phi)$ satisfies the equation

$$
x(n+1)=\sum_{k=0}^{n} Q(n-k) x(k)+p(n), \quad n \in \mathbf{Z}^{+} .
$$

As seen in the proof of the latter part of Theorem 1, the solution of Eq. (7) is given by the formula

$$
x(n)=R(n) x(0)+\sum_{k=0}^{n-1} R(n-k-1) p(k), \quad n \in \mathbf{Z}^{+} .
$$

Thus $x(\cdot ; \phi)$ is expressed as

$$
x(n ; \phi)=R(n) \phi(0)+\sum_{k=0}^{n-1} R(n-k-1)\left(\sum_{j=-\infty}^{-1} Q(k-j) \phi(j)\right), \quad n \in \mathbf{Z}^{+} .
$$


Now, we say that the zero solution of Eq. (2) is (globally) exponentially asymptotically stable, if there exist positive constants $K$ and $\alpha$ with the property that

$$
|x(n ; \phi)| \leq K e^{-\alpha n}\|\phi\|, \quad\left(\forall n \in \mathbf{Z}^{+}, \forall \phi \in \mathcal{B}^{\gamma}\right) .
$$

THEOREM 2. The zero solution of Eq. (2) is exponentially asymptotically stable if and only if the fundamental solution $R(n)$ of $E q$. (2) decays exponentially.

Consequently, we get the following relation: If the condition (4) holds true, then the zero solution of Eq. (2) is exponentially asymptotically stable; conversely, in case where $Q(j), j=0,1,2, \ldots$ are compact operators, if the zero solution of $E q$. (2) is exponentially asymptotically stable, then the condition (4) holds true.

Proof. The second part of the theorem is a direct consequence of the first part of the theorem and Theorem 1.

Now we will establish the first part of the theorem. Assume that $R(n)$ decays exponentially. Then there exist positive constants $M$ and $\epsilon$ with $\epsilon \in(0, \gamma)$ such that

$$
\|R(n)\| \leq M e^{-\epsilon n}, \quad n \in \mathbf{Z}^{+} .
$$

Then it follows from (9) that

$$
\begin{aligned}
|x(n ; \phi)| & \leq\|R(n)\||\phi(0)|+\sum_{k=0}^{n-1}\|R(n-k-1)\|\left(\sum_{j=-\infty}^{-1}\|Q(k-j)\||\phi(j)|\right) \\
& \leq M e^{-\epsilon n}\|\phi\|+\sum_{k=0}^{n-1} M e^{-\epsilon(n-k-1)}\left(l\|\phi\| e^{-\gamma k}\right) \\
& \leq M e^{-\epsilon n}\|\phi\|\left(1+\frac{l e^{\epsilon}}{1-e^{\epsilon-\gamma}}\right), \quad n \in \mathbf{Z}^{+}
\end{aligned}
$$

which shows the exponential asymptotic stability of the zero solution of Eq. (2).

Conversely, assume that the zero solution of Eq. (2) is exponentially asymptotically stable. For any $x \in X, R(\cdot) x$ is a solution of Eq. (2) through $(0, \phi)$ with $\phi(0)=x$ and $\phi(\theta) \equiv 0$ for $\theta=-1,-2, \ldots$. Hence it follows that

$$
|R(n) x| \leq K e^{-\alpha n}\|\phi\|=K e^{-\alpha n}|x|, \quad n \in \mathbf{Z}^{+},
$$

and hence

$$
\|R(n)\| \leq K e^{-\alpha n}, \quad n \in \mathbf{Z}^{+} .
$$

Thus $R(\cdot)$ decays exponentially. This completes the proof of the theorem.

For any $\phi \in \mathcal{B}^{\gamma}$ and $m \in \mathbf{Z}^{+}$, we set

$$
V(m) \phi=x_{m}(\phi)
$$


where $x_{m}$ denotes an element in $\mathcal{B}^{\gamma}$ defined by

$$
x_{m}(\theta)=x(m+\theta), \quad \theta \in \mathbf{Z}^{-} .
$$

We call $V(m)$ the solution operator of Eq. (2). The operator $V(m)$ is given by the formula

$$
\{V(m) \phi\}(\theta)= \begin{cases}\phi(m+\theta) & (\theta \leq-m), \\ R(m+\theta) \phi(0) & \\ +\sum_{r=0}^{m+\theta-1} R(m+\theta-r-1)\left(\sum_{j=-\infty}^{-1} Q(r-j) \phi(j)\right) & (-m \leq \theta \leq 0) .\end{cases}
$$

By this formula we see that $V(m)$ is a bounded linear operator on $\mathcal{B}^{\gamma}$. Furthermore, one can see that $V(m)$ satisfies the semigroup property:

$$
V(0)=I, \quad V(m) V(n)=V(m+n) \quad(\forall m, n \geq 0) .
$$

Corollary 1. Assume that the condition (4) holds true. Then $V(n)$ decays exponentially, that is,

$$
\exists K_{0}>0, \beta>0:\|V(n)\| \leq K_{0} e^{-\beta n}, \quad n \in \mathbf{Z}^{+} .
$$

Proof. By virtue of Theorem 2, the zero solution of Eq. (2) is exponentially asymptotically stable. Then there exist some positive constants $K$ and $\alpha$. with $K \geq 1$ and $\alpha<\gamma$ such that for any $\phi \in \mathcal{B}^{\gamma}, x(n):=x(n, \phi)$ satisfies

$$
|x(n)| \leq K e^{-\alpha n}\|\phi\|, \quad n \in \mathbf{Z}^{+} .
$$

Therefore it follows that

$$
\begin{aligned}
\|V(n) \phi\| & =\max \left(\sup _{-n \leq \theta \leq 0}|x(n+\theta)| e^{\gamma \theta}, \sup _{\theta \leq-n}|x(n+\theta)| e^{\gamma \theta}\right) \\
& \leq \max \left(\sup _{-n \leq \theta \leq 0} K e^{-\alpha(n+\theta)} e^{\gamma \theta}\|\phi\|, \sup _{\theta \leq-n}|\phi(n+\theta)| e^{\gamma \theta}\right) \\
& \leq\|\phi\| \max \left(K e^{-\alpha n}, e^{-\gamma n}\right) \\
& =K e^{-\alpha n}\|\phi\|,
\end{aligned}
$$

and hence

$$
\|V(n)\| \leq K e^{-\alpha n}, \quad n \in \mathbf{Z}^{+} .
$$

Thus $V(n)$ decays exponentially. 


\section{Volterra difference equations induced by differential equations with piecewise continuous delays}

One of the ways of deriving Volterra difference equations on a Banach space is based on some abstract differential equations with piecewise continuous delays. In fact, in [10] some differential equations on a Banach space is derived from some differential equations with piecewise continuous delays on a Banach space. In what follows, following the idea in [10], we explain how Volterra difference equations on a Banach space are canonically induced by some abstract differential equations with piecewise continuous delays. Moreover, we analyze the spectrum of the characteristic operator for the induced Volterra difference equations to obtain a condition under which some results established in the previous section are applicable.

In what follows, we employ the notation [ $\cdot]$ to denote the Gaussian symbol, and consider a differential equation

$$
\dot{u}(t)=A u(t)+\sum_{k=0}^{\infty} B(k) u([t-k]), \quad t \geq 0
$$

on a Banach space $X$, which contains piecewise continuous delays $t-[t-k], k=$ $0,1,2, \ldots$. Here and hereafter, we assume that $A$ is the inifinitesimal generator of a strongly continuous semigroup $T(t), t \geq 0$, of bounded linear operators on $X$, and $B(k), k=0,1,2, \ldots$, are bounded linear operators on $X$ such that

$$
\sum_{k=0}^{\infty}\|B(k)\| e^{\gamma k}<\infty
$$

for some $\gamma>0$. A function $u:\left(\mathbf{Z}^{-} \cup[0, \infty)\right) \rightarrow X$ such that $u_{0} \in \mathcal{B}^{\gamma}$ (that is, $\left.\sup _{\theta \in \mathbf{Z}^{-}}|u(\theta)| e^{\gamma \theta}<\infty\right)$ is called a (mild) solution of Eq. (10) on $[0, \infty)$, if $u$ is continuous on $[0, \infty)$, and it satisfies the following relation

$$
u(t)=T(t-\sigma) u(\sigma)+\int_{\sigma}^{t} T(t-s)\left(\sum_{k=0}^{\infty} B(k) u([s-k])\right) d s, \quad t \geq \sigma \geq 0 .
$$

In case when $n \leq t<n+1$ for some nonnegative integer $n$, the above relation yields that

$$
\begin{aligned}
u(t) & =T(t-n) u(n)+\int_{n}^{t} T(t-s)\left(\sum_{k=0}^{\infty} B(k) u([s-k])\right) d s \\
& =T(t-n) u(n)+\sum_{k=0}^{\infty}\left(\int_{n}^{t} T(t-s) B(k) u(n-k) d s\right) .
\end{aligned}
$$


Letting $t \rightarrow n+1$ in this equation, we get Volterra difference equation

$$
u(n+1)=\sum_{k=0}^{\infty} Q(k) u(n-k), \quad n \in \mathbf{Z}^{+},
$$

where $Q(k), k \in \mathbf{Z}^{+}$, are bounded linear operators on $X$ defined by

$$
\begin{aligned}
& Q(0) x=T(1) x+\int_{0}^{1} T(\tau) B(0) x d \tau \\
& Q(k) x=\int_{0}^{1} T(\tau) B(k) x d \tau, k=1,2, \ldots
\end{aligned}
$$

for $x \in X$.

Conversely, if $u$ satisfies Eq. (12) with $\sup _{\theta \in \mathbf{Z}^{-}}|u(\theta)| e^{\gamma \theta}<\infty$, then the function $u$ extended to non-integers $t$ by the relation

$$
u(t)=T(t-n) u(n)+\sum_{k=0}^{\infty}\left(\int_{n}^{t} T(t-s) B(k) u(n-k) d s\right),
$$

$n<t<n+1, n \in \mathbf{Z}^{+}$, is a (mild) solution of Eq. (10). Thus, some abstract differential equations such as Eq. (10) lead to Volterra difference equations on $X$. Sometimes, we call Eq. (12) the induced Volterra difference equation of Eq. (10).

A strongly continuous semigroup $T(t)$ on $X$ is said to be compact whenever $T(t)$ is a compact operator on $X$ for $t>0$. It is known [8] that if the semigroup $T(t)$ is compact, then $T(t)$ is continuous in $t>0$ with respect to the operator norm.

Proposition 1. If $T(t)$ is a compact semigroup on $X$ and $B \in \mathcal{L}(X)$, then the operator $Q \in \mathcal{L}(X)$ defined by the relation

$$
Q x=\int_{0}^{1} T(\tau) B x d \tau, \quad x \in X
$$

is compact.

Proof. For any $\epsilon \in(0,1)$ we set

$$
Q_{\epsilon}=\int_{\epsilon}^{1} T(\tau) B d \tau
$$

Then $Q_{\epsilon} \in \mathcal{L}(X)$, and moreover $Q_{\epsilon}$ is compact because of $Q_{\epsilon}=T(\epsilon / 2) \int_{\epsilon}^{1} T(\tau-$ $(\epsilon / 2)) B d \tau$. Furthermore, we get

$$
\left|\left(Q_{\epsilon}-Q\right) x\right|=\left|\int_{0}^{\epsilon} T(\tau) B x d \tau\right| \leq \epsilon C\|B\||x|, \quad x \in X
$$

where $C:=\sup _{0 \leq t \leq 1}\|T(t)\|(<\infty)$. Hence $\left\|Q_{\epsilon}-Q\right\| \leq \epsilon C\|B\| \rightarrow 0$ as $\epsilon \rightarrow+0$, and consequently $Q$ is a compact operator. 
By virtue of this proposition, $Q(k), k \in \mathbf{Z}^{+}$, defined by the relation (13) are compact operators on $X$ whenever $T(t)$ is a compact semigroup on $X$. Moreover, it follows from (11) that $Q(k), k \in \mathbf{Z}^{+}$satisfy the condition

$$
\sum_{k=0}^{\infty}\|Q(k)\| e^{\gamma k}<\infty
$$

In what follows, we restrict our consideration to the case where $B(k), k \in \mathbf{Z}^{+}$ are scalar, that is, $B(k) \equiv b(k) I, k \in \mathbf{Z}^{+}$, for some $b(k) \in \mathbf{C}$, and investigate the spectrum of the characteristic operator $z I-\tilde{Q}(z):=z I-\sum_{k=0}^{\infty} Q(k) z^{-k}$ of Eq. (12).

THEOREM 3. Let $T(t)$ be a compact semigroup on $X$, and assume that $B(k) \equiv b(k) I, k \in \mathbf{Z}^{+}$, where $b(k)$ is a scalar function satisfying $\sum_{k=0}^{\infty}|b(k)| e^{\gamma k}<$ $\infty$ for some positive constant $\gamma$. Then the spectrum of the characteristic operator $z I-\tilde{Q}(z)$ with $|z| \geq 1$ of Eq. (12) is given by

$$
\sigma(z I-\tilde{Q}(z))=\left(\{z\} \cup\left\{z-e^{\nu}-\tilde{b}(z) \int_{0}^{1} e^{\nu \tau} d \tau \mid \nu \in \sigma(A)\right\}\right)
$$

Proof. We first claim that

$$
\lim _{n \rightarrow \infty}\left\|\frac{1}{n} \sum_{k=1}^{n}\{T(1 / n)\}^{k}-\int_{0}^{1} T(\tau) d \tau\right\|=0 .
$$

Indeed, for any $x \in X$ it follows that

$$
\begin{aligned}
& \left|\left(\int_{0}^{1} T(\tau) d \tau\right) x-\left(\frac{1}{n} \sum_{k=1}^{n}\{T(1 / n)\}^{k}\right) x\right| \\
& =\left|\left(\int_{0}^{1} T(\tau) x d \tau\right)-\left(\frac{1}{n} \sum_{k=1}^{n} T(k / n) x\right)\right| \\
& =\left|\sum_{k=1}^{n}\left(\int_{\frac{k-1}{n}}^{\frac{k}{n}} T(\tau) x d \tau-\int_{\frac{k-1}{n}}^{\frac{k}{n}} T(k / n) x d \tau\right)\right| \\
& \quad \leq \sum_{k=1}^{n} \int_{\frac{k-1}{n}}^{\frac{k}{n}}\|T(\tau)-T(k / n)\| d \tau|x| .
\end{aligned}
$$

Hence we get

$$
\left\|\frac{1}{n} \sum_{k=1}^{n}\{T(1 / n)\}^{k}-\int_{0}^{1} T(\tau) d \tau\right\| \leq \sum_{k=1}^{n} \int_{\frac{k-1}{n}}^{\frac{k}{n}}\|T(\tau)-T(k / n)\| d \tau .
$$


For any $\epsilon>0$, take a positive constant $\eta_{0}, \eta_{0}<1 / 2$, such that $2 C \eta_{0}<\epsilon / 3$, where $C:=\sup \{\|T(t)\|: 0 \leq t \leq 1\}$. Recall that $T(t)$ is continuous in $t \in\left[\eta_{0}, 1\right]$ with respect to the operator norm. Then there exists a natural number $n_{0}$ satisfying $n_{0}>1 / \eta_{0}$ and $\sup \left\{\|T(\tau)-T(s)\|: \eta_{0} \leq s \leq \tau \leq 1,|\tau-s| \leq 1 / n_{0}\right\}<\epsilon / 3$. Let $n \geq n_{0}$. Putting $\left[n \eta_{0}\right]+1=k_{0}$, we get $k_{0} \geq 2$ and $\left(k_{0}-1\right) / n \leq \eta_{0}<1 / 2$; hence $k_{0}<n$. Note that $\eta_{0}<k_{0} / n$. Then

$$
\begin{aligned}
\| & \frac{1}{n} \sum_{k=1}^{n}\{T(1 / n)\}^{k}-\int_{0}^{1} T(\tau) d \tau \| \\
& \leq \sum_{k=1}^{k_{0}} \int_{\frac{k-1}{n}}^{\frac{k}{n}}\|T(\tau)-T(k / n)\| d \tau+\sum_{k=k_{0}+1}^{n} \int_{\frac{k-1}{n}}^{\frac{k}{n}}\|T(\tau)-T(k / n)\| d \tau \\
& \leq \frac{2 C k_{0}}{n}+\sup \left\{\|T(\tau)-T(s)\|: \eta_{0} \leq s \leq \tau \leq 1,|\tau-s| \leq 1 / n\right\} \\
& <2 C\left(\eta_{0}+\frac{1}{n}\right)+\frac{\epsilon}{3}<\epsilon .
\end{aligned}
$$

This proves the claim (15).

Now, set $S=\{T(t): 0 \leq t \leq 1\}$. Since $S$ is commutative, $\mathcal{A}:=\Gamma(\Gamma(S))$ is a commutative Banach algebra containing $S$, see [9, Theorem 11.22]. Here, for any subset $\Omega$ of $\mathcal{L}(X), \Gamma(\Omega)$ denotes the centralizer of $\Omega$; that is,

$$
\Gamma(\Omega)=\{v \in \mathcal{L}(X): v w=w v \text { for every } w \in \Omega\} .
$$

Let $\Delta$ be the maximal ideal space of $\mathcal{A}$. Let us denote by $\hat{a}$ the Gelfand transform of $a \in \mathcal{A}$. It is known [9, pp. 268-270] that $\hat{a}$ is a function from $\Delta$ (which is equipped with the Gelfand topology) into $\mathbf{C}$ with the properties that the range of $\hat{a}$ is the spectrum $\sigma(a)$ of $a$ and that

$$
\|\hat{a}\|_{\infty} \leq\|a\|, \quad a \in \mathcal{A},
$$

where $\|\hat{a}\|_{\infty}$ is the maximum of $|\hat{a}(\xi)|$ on $\Delta$. Moreover, the Gelfand transform is a homomorphism mapping $\mathcal{A}$ into a subspace of $C(\Delta ; \mathbf{C})$, the space of all the complex valued continuous functions on $\Delta$. Let $|z| \geq 1$, and put

$$
a=z I-\tilde{Q}(z)=z I-T(1)-\left(\int_{0}^{1} T(\tau) d \tau\right) \tilde{b}(z)
$$

and

$$
a_{n}=z I-W^{n}-\left(\frac{1}{n} \sum_{k=1}^{n} W^{k}\right) \tilde{b}(z)
$$


for each $n=1,2, \ldots$, where $W:=T(1 / n)$. Then $\left\{a, a_{1}, a_{2}, \ldots\right\} \subset \mathcal{A}$, and by (15) we get

$$
\left\|\widehat{\left(a_{n}\right)}-\hat{a}\right\|_{\infty} \leq\left\|a_{n}-a\right\|=\left\|\frac{1}{n} \sum_{k=1}^{n}\{T(1 / n)\}^{k}-\int_{0}^{1} T(\tau) d \tau\right\||\tilde{b}(z)| \rightarrow 0
$$

as $n \rightarrow \infty$. Thus

$$
\lim _{n \rightarrow \infty} \widehat{\left(a_{n}\right)}(\xi)=\hat{a}(\xi), \quad \xi \in \Delta .
$$

Observe that $\widehat{\left(a_{n}\right)}(\xi)=z-(\hat{W}(\xi))^{n}-\frac{1}{n} \sum_{k=1}^{n}(\hat{W}(\xi))^{k} \tilde{b}(z)$. Since the operator $T(1 / n)$ is compact, the Riesz-Schauder theorem implies that $\sigma(T(1 / n))=$ $P_{\sigma}(T(1 / n)) \cup\{0\}$. Also, it follows from [8, Theorems 2.2.3-2.2.4] that

$$
\exp ((1 / n) \sigma(A)) \subset \sigma(T(1 / n)), \quad P_{\sigma}(T(1 / n)) \cup\{0\}=\exp \left((1 / n) P_{\sigma}(A)\right) \cup\{0\} .
$$

Therefore we get $\sigma(W)=\sigma(T(1 / n))=\exp \left((1 / n) P_{\sigma}(A)\right) \cup\{0\}$. From these observations, we see that the range of $\widehat{\left(a_{n}\right)}$ is identical with the set

$$
\{z\} \cup\left(\left\{z-e^{\nu}-\frac{1}{n} \sum_{k=1}^{n} e^{(k / n) \nu} \tilde{b}(z) \mid \nu \in \sigma(A)\right\}\right) .
$$

Note that $\lim _{n \rightarrow \infty}(1 / n) \sum_{k=1}^{n} e^{(k / n) \nu}=\int_{0}^{1} e^{\nu \tau} d \tau$. Therefore, combining this fact with (16) we conclude that the set in the right hand side of (14) is identical with the range of $\hat{a}$ which is equal to $\sigma(a)=\sigma(z I-\tilde{Q}(z))$. This completes the proof.

Combining Theorem 3 with Theorem 2, we get the following result:

Corollary 2. Let $T(t)$ be a compact semigroup on $X$, and assume that $B(k) \equiv b(k) I, \quad k \in \mathbf{Z}^{+}$, where $b(k)$ is a scalar function satisfying $\sum_{k=0}^{\infty}|b(k)| e^{\gamma k}<$ $\infty$ for some positive constant $\gamma$. Then the following three statements are equivalent:

(i) The condition (4) for Eq. (12) holds true:

(ii) The zero solution of Eq. (12) is exponentially asymptotically stable:

(iii) $z \neq e^{\nu}+\tilde{b}(z) \int_{0}^{1} e^{\nu \tau} d \tau, \quad(\forall|z| \geq 1, \nu \in \sigma(A))$.

\section{Stability analysis in some population models}

In this section we consider a scalar partial differential equation with piecewise continuous delays

$$
\begin{array}{r}
\frac{\partial v}{\partial t}(t, x)=\frac{\partial^{2} v}{\partial x^{2}}(t, x)+\delta v(t, x)\left(1-(1 / K) \sum_{j=0}^{\infty} c(j) v([t-j], x)\right) \\
t>0,0<x<\pi
\end{array}
$$


with the Neumann boundary condition

$$
\frac{\partial v}{\partial x}(t, 0)=\frac{\partial v}{\partial x}(t, \pi)=0, \quad t>0
$$

which describes the population dynamics in a single species taking diffusion effects into consideration. Equations with piecewise continuous delays modeling the population dynamics without taking diffusion effects into consideration are treated in many literatures such as $[1,2,5,6,7]$ and the references therein. In what follows, as an application of the results obtained in the previous sections, we investigate stability properties of the positive constant solution of (17)-(18). Throughout this section, without stating explicitly from now on, we assume that $\delta$ and $K$ are positive constants, and $c(j), j \in \mathbf{Z}^{+}$, is a nonnegative sequence such that $\sum_{j=0}^{\infty} c(j)=1$ and $\sum_{j=0}^{\infty} c(j) e^{\gamma j}<\infty$ for some positive constant $\gamma$. Obviously, $v(t, x) \equiv K$ is a solution of (17)-(18). In order to investigate the stability of the equilibrium $v \equiv K$, we set $u=v-K$. Then (17)-(18) is transformed to the equation

$$
\frac{\partial u}{\partial t}(t, x)=\frac{\partial^{2} u}{\partial x^{2}}(t, x)-\frac{\delta}{K}(u(t, x)+K) \sum_{j=0}^{\infty} c(j) u([t-j], x), \quad t>0,0<x<\pi
$$

and

$$
\frac{\partial u}{\partial x}(t, 0)=\frac{\partial u}{\partial x}(t, \pi)=0, \quad t>0 .
$$

Take $X=C([0, \pi] ; \mathbf{R})$ and set $A \xi=\xi^{\prime \prime}$ for $\xi \in \mathcal{D}(A):=\left\{\xi \in C^{2}([0, \pi] ; \mathbf{R}): \xi^{\prime}(0)=\right.$ $\left.\xi^{\prime}(\pi)=0\right\} . A$ generates a compact strongly continuous semigroup $T(t), t \geq 0$, on $X=C([0, \pi] ; \mathbf{R})$. In fact, $T(t)$ is given by the formula

$$
T(t) \xi \sim \sum_{n=0}^{\infty} e^{-n^{2} t} a_{n} \cos n x, \quad t \geq 0
$$

for any $\xi \in X$ with the Fourier expansion $\sum_{n=0}^{\infty} a_{n} \cos n x$, and moreover we have $\sigma(A)=\left\{-n^{2}: n=0,1,2, \ldots\right\}$. Then (19)-(20) can be set up as a differential equation with piecewise continuous delays on the Banach space $X=C([0, \pi] ; \mathbf{R})$ for which the linearized equation is of the form such as Eq. (10);

$$
\dot{u}(t)=A u(t)+\sum_{k=0}^{\infty} B(k) u([t-k]), \quad t \geq 0,
$$

where $B(n) \equiv-\delta c(n) I$. In the following, we will derive the following result on the stability of the constant solution of (17)-(18): 
Theorem 4. Suppose that the following condition holds true:

$$
z \neq 1-\delta \tilde{c}(z) \quad \text { and } z \neq e^{-n^{2}}+\frac{\delta\left(e^{-n^{2}}-1\right)}{n^{2}} \tilde{c}(z), \quad(n=1,2, \ldots,|z| \geq 1) .
$$

Then the constant solution $v(t, x) \equiv K$ of (17)-(18) is exponentially asymptotically stable.

The condition (21) is satisfied in the case that $0<\delta<2$ and $c(0)=1$ with $c(n)=0, n=1,2, \ldots$, or in the case that $0<\delta<6$ and $c(n)=2^{-n-1}, n \in \mathbf{Z}^{+}$. Indeed, in case of $c(n)=2^{-n-1}, n \in \mathbf{Z}^{+}$, we get $\tilde{c}(z)=z /(2 z-1)$ for $|z| \geq 1$, and an elementary calculation shows that the quadratic equation $2 z^{2}+(\delta-3) z+1=0$, which is a transformation of the equation $z=1-\delta \tilde{c}(z)$, has no roots $\lambda$ such that $|\lambda| \geq 1$ if and only if $0<\delta<6$. Also, one can easily check that if $0<\delta<6$, then the equations $z=e^{-n^{2}}+\delta \tilde{c}(z)\left(e^{-n^{2}}-1\right) / n^{2}, n=1,2, \ldots$, have no roots $\lambda$ such that $|\lambda| \geq 1$. This observation leads to the fact that the condition (21) is satisfied in the case that $0<\delta<6$ and $c(n)=2^{-n-1}, n \in \mathbf{Z}^{+}$.

In case of $c(0)=1$ with $c(n)=0, n=1,2, \ldots$, we get $\tilde{c}(z) \equiv 1$ for $|z| \geq 1$, and in a similar way one can certify the condition (21) under the assumption that $0<\delta<2$.

Before proving the theorem we prepare some results on the solution operators of the induced Volterra difference equation (12) and the original differential equation with piecewise continuous delays

$$
\dot{u}(t)=A u(t)+\sum_{k=0}^{\infty} B(k) u([t-k]), \quad t \in \mathbf{R}^{+}:=[0, \infty) .
$$

Eq. (22) can be viewed as an abstract functional differential equation

$$
\dot{u}(t)=A u(t)+F\left(t, u_{t}\right), \quad t \geq 0,
$$

where $u_{t}$ is the function defined by $u_{t}(\theta)=u(t+\theta)$ for $\theta \in \mathbf{R}^{-}:=(-\infty, 0]$, and $F(t, \phi):=\sum_{k=0}^{\infty} B(k) \phi([t-k]-t)$ is an operator from $\mathbf{R}^{+} \times \mathcal{C}^{\gamma}$ into $X$ which is 1-periodic in $t$, bounded and linear in $\phi$, and is continuous in $t$ at any non-integral values of $t$. Here and hereafter, $\mathcal{C}^{\gamma}$ is the Banach space given by

$$
\begin{array}{r}
\mathcal{C}^{\gamma}=\left\{\phi: \mathbf{R}^{-} \rightarrow X \mid \phi(\theta) e^{\gamma \theta} \text { is uniformly continuous on } \mathbf{R}^{-}\right. \\
\text {and } \left.\sup _{\theta \in \mathbf{R}^{-}}|\phi(\theta)| e^{\gamma \theta}<\infty\right\}
\end{array}
$$

with norm $\|\phi\|=\sup _{\theta \in \mathbf{R}^{-}}|\phi(\theta)| e^{\gamma \theta}$ for $\phi \in \mathcal{C}^{\gamma}$.

For any $(\sigma, \phi) \in \mathbf{R}^{+} \times \mathcal{C}^{\gamma}$ there exists uniquely a function $u: \mathbf{R} \rightarrow X$ such 
that $u \in C([\sigma, \infty) ; X), u_{\sigma}(\theta) \equiv \phi(\theta)$ for $\theta \in \mathbf{R}^{-}$, and

$$
u(t)=T(t-\sigma) \phi(0)+\int_{\sigma}^{t} T(t-s)\left(\sum_{k=0}^{\infty} B(k) u([s-k])\right) d s, \quad t \geq \sigma .
$$

We call such a function $u$ the (mild) solution of Eq. (22) through $(\sigma, \phi)$, and denote it by $u(\cdot, \sigma, \phi ; 0)$.

For any $t \geq \sigma \geq 0$, let us denote by $U(t, \sigma)$ the solution operator of Eq. (22); that is,

$$
U(t, \sigma) \phi=u_{t}(\sigma, \phi ; 0), \quad t \geq \sigma, \phi \in \mathcal{C}^{\gamma} .
$$

$U(t, \sigma)$ is a bounded linear operator on $\mathcal{C}^{\gamma}$. It is obvious that $U(t, t)=I$.

Proposition 2. The followings assertions hold true:

(i) $U(t, \tau) U(\tau, \sigma)=U(t, \sigma), \quad t \geq \tau \geq \sigma$;

(ii) $U(t, \sigma)$ is 1-periodic; that is, $U(t+1, \sigma+1)=U(t, \sigma), \quad t \geq \sigma$;

(iii) $\sup _{0 \leq \sigma \leq t \leq[\sigma]+1}\|U(t, \sigma)\|=: D<\infty$.

Proof. (i) Set $w(t)=u\left(t, \tau, u_{\tau}(\sigma, \phi ; 0) ; 0\right)$ for $t \in \mathbf{R}$. If $t \leq \tau$, then

$$
w(t)=\left(u_{\tau}(\sigma, \phi ; 0)\right)(t-\tau)=u(t, \sigma, \phi ; 0) .
$$

Hence, for any $\mu \in[\sigma, \tau]$ we get

$$
\begin{aligned}
w(\mu) & =u(\mu, \sigma, \phi ; 0) \\
& =T(\mu-\sigma) \phi(0)+\int_{\sigma}^{\mu} T(\mu-s)\left(\sum_{k=0}^{\infty} B(k) u([s-k], \sigma, \phi ; 0)\right) d s \\
& =T(\mu-\sigma) \phi(0)+\int_{\sigma}^{\mu} T(\mu-s)\left(\sum_{k=0}^{\infty} B(k) w([s-k])\right) d s
\end{aligned}
$$

On the one hand, for any $\mu \geq \tau$ we get

$$
\begin{gathered}
w(\mu)=T(\mu-\tau) u(\tau, \sigma, \phi ; 0)+\int_{\tau}^{\mu} T(\mu-s)\left(\sum_{k=0}^{\infty} B(k) w([s-k])\right) d s \\
=T(\mu-\tau)\left\{T(\tau-\sigma) \phi(0)+\int_{\sigma}^{\tau} T(\tau-s)\left(\sum_{k=0}^{\infty} B(k) u([s-k], \sigma, \phi ; 0)\right) d s\right\} \\
+\int_{\tau}^{\mu} T(\mu-s)\left(\sum_{k=0}^{\infty} B(k) w([s-k])\right) d s
\end{gathered}
$$




$$
\begin{aligned}
=T(\mu-\sigma) \phi(0)+\int_{\sigma}^{\tau} T(\mu-s)\left(\sum_{k=0}^{\infty} B(k) w([s-k])\right) d s \\
\quad+\int_{\tau}^{\mu} T(\mu-s)\left(\sum_{k=0}^{\infty} B(k) w([s-k])\right) d s \\
=T(\mu-\sigma) \phi(0)+\int_{\sigma}^{\mu} T(\mu-s)\left(\sum_{k=0}^{\infty} B(k) w([s-k])\right) d s .
\end{aligned}
$$

It follows from these observations that $w$ is a solution of Eq. (22) with $w_{\sigma}=\phi$, and hence $w \equiv u(\sigma, \phi ; 0)$, or $u\left(\cdot, \tau, u_{\tau}(\sigma, \phi ; 0) ; 0\right) \equiv u(\cdot, \sigma, \phi ; 0)$. Consequently, we get

$$
U(t, \tau) U(\tau, \sigma) \phi=u_{t}\left(\tau, u_{\tau}(\sigma, \phi ; 0) ; 0\right)=u_{t}(\sigma, \phi ; 0)=U(t, \sigma) \phi
$$

which completes the proof of (i).

(ii) $v(t):=u(t+1, \sigma+1, \phi ; 0)$ satisfies $v_{\sigma}=\phi$ and

$$
\begin{aligned}
v(t) & =T(t-\sigma) \phi(0)+\int_{\sigma+1}^{t+1} T(t+1-s)\left(\sum_{k=0}^{\infty} B(k) u([s-k] ; \sigma+1, \phi ; 0)\right) d s \\
& =T(t-\sigma) \phi(0)+\int_{\sigma}^{t} T(t-\tau)\left(\sum_{k=0}^{\infty} B(k) u([\tau+1-k] ; \sigma+1, \phi ; 0)\right) d \tau \\
& =T(t-\sigma) \phi(0)+\int_{\sigma}^{t} T(t-\tau)\left(\sum_{k=0}^{\infty} B(k) v([\tau-k])\right) d \tau
\end{aligned}
$$

for any $t \geq \sigma$. Therefore we get $v(\cdot)=u(\cdot, \sigma, \phi ; 0)$, or $u_{t+1}(\sigma+1, \phi ; 0)=u_{t}(\sigma, \phi ; 0)$, which completes the proof of (ii).

(iii) Let $0 \leq \sigma \leq t \leq 1$. Then

$$
\begin{aligned}
|u(t, \sigma, \phi ; 0)| & =\left|T(t-\sigma) \phi(0)+\int_{\sigma}^{t} T(t-s)\left(\sum_{k=0}^{\infty} B(k) u(-k, \sigma, \phi ; 0)\right) d s\right| \\
& \leq C|\phi(0)|+C\left(\sum_{k=0}^{\infty}\|B(k)\||\phi(-k-\sigma)|\right) \\
& \leq C\|\phi\|\left(1+\sum_{k=0}^{\infty}\|B(k)\| e^{\gamma(k+\sigma)}\right) \\
& \leq C\|\phi\|\left(1+L e^{\gamma}\right)
\end{aligned}
$$

where $C:=\sup _{0 \leq \tau \leq 1}\|T(\tau)\|$ and $L:=\sum_{k=0}^{\infty}\|B(k)\| e^{\gamma k}$. Thus $\|U(t, \sigma)\| \leq C(1+$ $L e^{\gamma}$ ) for any $0 \leq \sigma \leq t \leq 1$. Then (iii) follows from this fact and the periodicity of $U(t, \sigma)$ by setting $D=C\left(1+L e^{\gamma}\right)$. 
Proposition 3. Assume that the zero solution of the Volterra difference equation induced by Eq. (22) is exponentially asymptotically stable. Then there exist positive constants $M \geq 1$ and $\alpha \in(0,1)$ such that

$$
\|U(t, \sigma)\| \leq M e^{-\alpha(t-\sigma)}, \quad t \geq \sigma \geq 0 .
$$

Proof. By the assumption, the solution operator $V(\cdot)$ of Eq. (12) satisfies the estimate

$$
\|V(n)\| \leq c e^{-\beta n}, \quad n \in \mathbf{N}^{+}
$$

for some constant $c \geq 1$ and $\beta \in(0,1)$. Set $M_{0}=D^{2} e^{2}$, where $D, D \geq 1$, is the constant whose existence was assured in the preceding proposition. If $\sigma \leq t \leq$ $[\sigma]+2$, then the estimate (23) with $M_{0}$ in place of $M$ is a direct consequence of the preceding proposition. We will establish the estimate (23) in case of $t>[\sigma]+2$. We may assume that $0 \leq \sigma<1$ and $n \leq t<n+1$ for some natural number $n \geq 2$. The preceding proposition yields that

$$
U(t, \sigma)=U(t, n) U(n, 1) U(1, \sigma)=U(t, n) U(n-1,0) U(1, \sigma) .
$$

Let $\Pi$ be a bounded linear operator from $C^{\gamma}$ into $B^{\gamma}$ defined by

$$
(\Pi \phi)(s)=\phi(s), \quad s \in \mathbf{Z}^{-}, \phi \in C^{\gamma} .
$$

It is easy to see that $\|\Pi\| \leq 1$. We assert that

$$
\Pi U(1,0)=V(1) \Pi \text {. }
$$

Indeed, for any $\phi \in \mathcal{C}^{\gamma}$ we get $u(1,0, \phi ; 0)=\sum_{k=0}^{\infty} Q(k) \phi(-k)$, and consequently $\Pi U(1,0) \phi=\Pi u_{1}(0, \phi ; 0)=V(1) \Pi \phi$, which shows that the assertion holds true. Therefore

$$
\Pi U(n-1,0)=\Pi\{U(1,0)\}^{n-1}=\{V(1)\}^{n-1} \Pi=V(n-1) \Pi .
$$

In particular, it follows that

$$
\begin{aligned}
\left\|\Pi u_{n}(\sigma, \phi ; 0)\right\| & =\left\|V(n-1) \Pi u_{1}(\sigma, \phi ; 0)\right\| \\
& \leq c e^{-\beta(n-1)}\|U(1, \sigma)\|\|\phi\| \\
& \leq e c D e^{-\beta n}\|\phi\| .
\end{aligned}
$$

Then, for any $m \in \mathbf{N}$ with $m \leq n$ and any $s$ with $[s]=m$ we have

$$
\begin{aligned}
|u(s, \sigma, \phi ; 0)|=\mid T(s-m) & u(m, \sigma, \phi ; 0) \\
& +\int_{m}^{s} T(s-\tau)\left(\sum_{k=0}^{\infty} B(k) u(m-k, \sigma, \phi ; 0)\right) d \tau \mid
\end{aligned}
$$




$$
\begin{aligned}
& \leq C(1+L)\left\|\Pi u_{m}(\sigma, \phi ; 0)\right\| \\
& \leq C(1+L) e c D e^{-\beta m}\|\phi\| \leq C(1+L) e^{2} c D e^{-\beta(s-\sigma)}\|\phi\|,
\end{aligned}
$$

where $C:=\sup _{0 \leq \tau \leq 1}\|T(\tau)\|$ and $L:=\sum_{k=0}^{\infty}\|B(k)\| e^{\gamma k}$. It follows from this observation that

$$
\begin{aligned}
\left\|u_{s}(\sigma, \phi ; 0)\right\| & =\max \left(\sup _{\sigma \leq \tau \leq s} e^{\gamma(\tau-s)}|u(\tau, \sigma, \phi ; 0)|, \sup _{\tau \leq 0} e^{\gamma(\tau+\sigma-s)}|\phi(\tau)|\right) \\
& \leq \max \left(\sup _{\sigma \leq \tau \leq s}|u(\tau, \sigma, \phi ; 0)|,\|\phi\|\right) \\
& \leq \max \left(1, C(1+L) e^{2} c D\right)\|\phi\|
\end{aligned}
$$

and hence

$$
\begin{aligned}
\left\|u_{t}(\sigma, \phi ; 0)\right\| & =\max \left(\sup _{-t / 2 \leq \theta \leq 0} e^{\gamma \theta}|u(t+\theta, \sigma, \phi ; 0)|, \sup _{\theta \leq-t / 2} e^{\gamma \theta}|u(t+\theta, \sigma, \phi ; 0)|\right) \\
& \leq \max \left(\sup _{t / 2 \leq s \leq t}|u(s, \sigma, \phi ; 0)|, \sup _{\tau \leq 0} e^{\gamma(\tau-(t / 2))}|u(t / 2+\tau, \sigma, \phi ; 0)|\right) \\
& \leq \sup _{t / 2 \leq s \leq t}|u(s, \sigma, \phi ; 0)|+e^{-\gamma t / 2}\left\|u_{t / 2}(\sigma, \phi ; 0)\right\| \\
& \leq C(1+L) e^{2} c D e^{-\beta(t / 2-\sigma)}\|\phi\|+e^{-\gamma t / 2} \max \left(1, C(1+L) e^{2} c D\right)\|\phi\| \\
& \leq\left(C(1+L) e^{2+\beta} c D+\max \left(1, C(1+L) e^{2} c D\right)\right) e^{-\alpha t}\|\phi\|
\end{aligned}
$$

where $\alpha=(1 / 2) \min (\beta, \gamma)$. Therefore, the estimate (23) holds with $M=M_{0}+$ $\left(C(1+L) e^{2+\beta} c D+\max \left(1, C(1+L) e^{2} c D\right)\right)$.

For any natural number $n$, define an $\mathcal{L}(X)$-valued function $\Gamma^{n}$ by

$$
\Gamma^{n}(\theta)=\left\{\begin{array}{cl}
(n \theta+1) I, & -1 / n \leq \theta \leq 0 \\
0, & \theta<-1 / n .
\end{array}\right.
$$

It is clear that if $x \in X$, then $\Gamma^{n} x \in \mathcal{C}^{\gamma}$ with $\left\|\Gamma^{n} x\right\|=|x|$.

Notice that if $t<\sigma$, then $\lim _{n \rightarrow \infty} u\left(t, \sigma, \Gamma^{n} x ; 0\right)=0$. For any $t \geq \sigma$ let us consider the limit of $u\left(t, \sigma, \Gamma^{n} x ; 0\right)=\left\{U(t, \sigma)\left(\Gamma^{n} x\right)\right\}(0)$ as $n \rightarrow \infty$. In case of $0 \leq \sigma \leq t \leq 1$, it follows that

$$
\begin{aligned}
u\left(t, \sigma, \Gamma^{n} x ; 0\right) & =T(t-\sigma) x+\int_{\sigma}^{t} T(t-s)\left(\sum_{k=0}^{\infty} B(k) u\left([s-k], \sigma, \Gamma^{n} x ; 0\right)\right) d s \\
& =T(t-\sigma) x+\int_{\sigma}^{t} T(t-s) B(0) u\left(0, \sigma, \Gamma^{n} x ; 0\right) d s
\end{aligned}
$$




$$
=T(t-\sigma) x+\int_{\sigma}^{t} T(t-s) B(0) \Gamma^{n}(-\sigma) x d s,
$$

and consequently $u\left(t, \sigma, \Gamma^{n} x ; 0\right)$ tends to $T(t-\sigma) x$ if $\sigma>0$, and $T(t) x+\int_{0}^{t} T(t-$ $s) B(0) x d s$ if $\sigma=0$. Generally, one can derive that for any $t, \sigma$ and $x \in X$, $u\left(t, \sigma, \Gamma^{n} x ; 0\right)$ tends to some element in $X$ (which we denote by $\left.r(t, \sigma) x\right)$ as $n \rightarrow \infty$ :

$$
\lim _{n \rightarrow \infty} u\left(t, \sigma, \Gamma^{n} x ; 0\right)=r(t, \sigma) x
$$

In fact, $r(t, \sigma)$ is a 1-periodic function satisfying $r(t, t)=I$ and $r(t, \sigma)=0$ for $t<\sigma$. Moreover, $r(t, \sigma) x$ for $t \geq \sigma$ with $0 \leq \sigma<1$ is given by the following relations:

$$
\begin{gathered}
r(t, \sigma) x=T(t-\sigma) x+\sum_{k=1}^{[t]-1} \int_{k}^{k+1} T(t-s)\left(\sum_{j=0}^{k-1} B(j) r(k-j, \sigma) x\right) d s \\
+\int_{[t]}^{t} T(t-s)\left(\sum_{j=0}^{[t]-1} B(j) r([t]-j, \sigma) x\right) d s, \quad 0<\sigma<1 ; \\
r(t, 0) x=T(t) x+\sum_{k=0}^{[t]-1} \int_{k}^{k+1} T(t-s)\left(\sum_{j=0}^{k} B(j) r(k-j, 0) x\right) d s \\
r(t, \sigma) x=\left\{\begin{array}{l}
T(t-\sigma) x, \quad 0<\sigma \leq t \leq 1, \sigma<1, \\
T(t) x+\int_{0}^{t} T(t-s) B(0) x d s, \quad \sigma=0 \leq t \leq 1,
\end{array}\right.
\end{gathered}
$$

where we used the convention $\sum_{0}^{-1}=0$. Consequently, $r(t, \sigma)$ is an $\mathcal{L}(X)$-valued function which is strongly continuous in $(t, \sigma), t \geq \sigma$, with $\sigma \neq$ integers. In particular, for any $X$-valued bounded function $h$ which is continuous except at integers, the function $r(t, s) h(s)$ is locally integrable in $s$.

Now, let us recall that $u(\cdot, \sigma, 0 ; h)$ is a solution of

$$
\dot{u}(t)=A u(t)+\sum_{k=0}^{\infty} B(k) u([t-k])+h(t)
$$

with $u_{\sigma}=0$. 
Proposition 4. Let $h$ be any $X$-valued bounded function which is continuous except at integers. Then the following relation holds:

$$
\int_{\sigma}^{t} r(t, s) h(s) d s=u(t, \sigma, 0 ; h), \quad t \geq \sigma \geq 0 .
$$

Proof. It suffices to certify the relation (25) in case of $0 \leq \sigma<1$. In case of $0 \leq \sigma \leq t \leq 1$, we have

$$
\begin{aligned}
u(t, \sigma, 0 ; h) & =\int_{\sigma}^{t} T(t-s)\left(\sum_{k=0}^{\infty} B(k) u([s-k], \sigma, 0 ; h)+h(s)\right) d s \\
& =\int_{\sigma}^{t} T(t-s) h(s) d s \\
& =\int_{\sigma}^{t} r(t, s) h(s) d s,
\end{aligned}
$$

as required. In order to establish the relation by induction, we assume that the relation holds true for all $t$ such that $\sigma \leq t \leq n$, and consider the case of $n<t \leq$ $n+1$. We get

$$
\begin{aligned}
& u(t, \sigma, 0 ; h) \\
& =\int_{\sigma}^{t} T(t-s) h(s) d s+\sum_{k=1}^{n-1} \int_{k}^{k+1} T(t-s)\left(\sum_{j=0}^{\infty} B(j) u([s-j], \sigma, 0 ; h)\right) d s \\
& +\int_{n}^{t} T(t-s)\left(\sum_{j=0}^{\infty} B(j) u([s-j], \sigma, 0 ; h)\right) d s \\
& =\int_{\sigma}^{t} T(t-s) h(s) d s+\sum_{k=1}^{n-1} \int_{k}^{k+1} T(t-s)\left(\sum_{j=0}^{k-1} B(j) u(k-j, \sigma, 0 ; h)\right) d s \\
& +\int_{n}^{t} T(t-s)\left(\sum_{j=0}^{n-1} B(j) u(n-j, \sigma, 0 ; h)\right) d s \\
& =\int_{\sigma}^{t} T(t-s) h(s) d s+\sum_{k=1}^{n-1} \int_{k}^{k+1} T(t-s)\left(\sum_{j=0}^{k-1} B(j) \int_{\sigma}^{k-j} r(k-j, \tau) h(\tau) d \tau\right) d s \\
& +\int_{n}^{t} T(t-s)\left(\sum_{j=0}^{n-1} B(j) \int_{\sigma}^{n-j} r(n-j, \tau) h(\tau) d \tau\right) d s
\end{aligned}
$$

where we used the assumption in the induction. Since $r(k-j, \tau)=0$ for $\tau>k-j$, 
we rewrite the integrals as

$$
\int_{\sigma}^{k-j} r(k-j, \tau) h(\tau) d \tau=\int_{\sigma}^{t} r(k-j, \tau) h(\tau) d \tau, \quad 0 \leq j<k \leq n .
$$

Therefore, by changing the order of integration we have

$$
\begin{aligned}
& u(t, \sigma, 0 ; h) \\
& =\int_{\sigma}^{t} T(t-\tau) h(\tau) d \tau+\sum_{k=1}^{n-1} \int_{k}^{k+1} T(t-s)\left(\sum_{j=0}^{k-1} B(j) \int_{\sigma}^{t} r(k-j, \tau) h(\tau) d \tau\right) d s \\
& +\int_{n}^{t} T(t-s)\left(\sum_{j=0}^{n-1} B(j) \int_{\sigma}^{t} r(n-j, \tau) h(\tau) d \tau\right) d s \\
& =\int_{\sigma}^{t} T(t-\tau) h(\tau) d \tau+\int_{\sigma}^{t} \sum_{k=1}^{n-1}\left(\int_{k}^{k+1} T(t-s) \sum_{j=0}^{k-1} B(j) r(k-j, \tau) h(\tau) d s\right) d \tau \\
& +\int_{\sigma}^{t}\left(\int_{n}^{t} T(t-s) \sum_{j=0}^{n-1} B(j) r(n-j, \tau) h(\tau) d s\right) d \tau \\
& =\int_{\sigma}^{t}\left\{T(t-\tau) h(\tau)+\sum_{k=1}^{[t]-1} \int_{k}^{k+1} T(t-s) \sum_{j=0}^{k-1} B(j) r(k-j, \tau) h(\tau) d s\right. \\
& \left.+\int_{[t]}^{t} T(t-s) \sum_{j=0}^{[t]-1} B(j) r([t]-j, \tau) h(\tau) d s\right\} d \tau
\end{aligned}
$$

We now assert that Formula (24) holds true for any $t$ and $\sigma$ with $t \geq \sigma \geq 0$ and $\sigma \neq$ integers. If the assertion is true, it follows from the above relation that $u(t, \sigma, 0 ; h)=\int_{\sigma}^{t} r(t, \tau) h(\tau) d \tau$, and hence the relation (25) holds true when $n<t \leq$ $n+1$. Therefore, the relation (25) holds true for any $t$ by induction on $n$.

In the remainder, we will certify the assertion. Let $t \geq \sigma \geq 0$ with $\sigma \neq$ integers. By virtue of Formula (24) and the periodicity of $r(t, \sigma)$, we have

$$
\begin{aligned}
& r(t, \sigma) x-T(t-\sigma) x \\
& =r(t-[\sigma], \sigma-[\sigma])-T(t-\sigma) x \\
& =\sum_{m=1}^{[t]-[\sigma]-1} \int_{m}^{m+1} T(t-[\sigma]-\nu) \sum_{j=0}^{m-1} B(j) r(m-j, \sigma-[\sigma]) x d \nu \\
& \quad+\int_{[t]-[\sigma]}^{t-[\sigma]} T(t-[\sigma]-\nu) \sum_{j=0}^{[t]-[\sigma]-1} B(j) r([t]-[\sigma]-j, \sigma-[\sigma]) x d \nu
\end{aligned}
$$




$$
\begin{gathered}
=\sum_{m=1}^{[t]-[\sigma]-1} \int_{m+[\sigma]}^{m+[\sigma]+1} T(t-s) \sum_{j=0}^{m-1} B(j) r(m-j, \sigma-[\sigma]) x d s \\
\quad+\int_{[t]}^{t} T(t-s) \sum_{j=0}^{[t]-[\sigma]-1} B(j) r([t]-j, \sigma) x d s \\
=\sum_{k=[\sigma]+1} \int_{k}^{k+1} T(t-s) \sum_{j=0}^{k-[\sigma]-1} B(j) r(k-j, \sigma) x d s \\
\quad+\int_{[t]}^{t} T(t-s) \sum_{j=0}^{[t]-[\sigma]-1} B(j) r([t]-j, \sigma) x d s .
\end{gathered}
$$

Observe that if $k-[\sigma] \leq j$, then $k-j \leq[\sigma]<\sigma$ and $r(k-j, \sigma)=0$. This observation leads to

$$
\begin{array}{r}
r(t, \sigma) x-T(t-\sigma) x=\sum_{k=[\sigma]+1}^{[t]-1} \int_{k}^{k+1} T(t-s) \sum_{j=0}^{k-1} B(j) r(k-j, \sigma) x d s \\
\quad+\int_{[t]}^{t} T(t-s) \sum_{j=0}^{[t]-1} B(j) r([t]-j, \sigma) x d s
\end{array}
$$

which proves the assertion, because $k \leq[\sigma]$ implies $k-j \leq k \leq[\sigma]<\sigma$ and $r(k-j, \sigma)=0$ for $j=0,1, \ldots, k-1$. This completes the proof of the proposition.

Proof of Theorem 4. In virtue of the condition (21), one can apply Corollary 2 with $b(n)=-\delta c(n)$ to conclude that the zero solution of the Volterra difference equation induced by Eq. (22) is exponentially asymptotically stable. Hence, by Corollary 1 and Proposition 3 we get the estimate $\|U(t, s)\| \leq M e^{-\alpha(t-s)}, t \geq s \geq 0$, for some constants $M \geq 1$ and $\alpha>0$. It follows that

$$
|r(t, s) x| \leq \limsup _{n \rightarrow \infty}\left\|U(t, s)\left(\Gamma^{n} x\right)\right\| \leq M e^{-\alpha(t-s)}|x|,
$$

and consequently

$$
\|r(t, s)\| \leq M e^{-\alpha(t-s)}, \quad t \geq s \geq 0 .
$$

Set $\eta:=K \alpha /\left(2 M \delta\left(1+l e^{\gamma}\right)\right)$, where $l:=\sum_{n=0}^{\infty} c(n) e^{\gamma n}$. Take any $(\sigma, \phi) \in \mathbf{R}^{+} \times \mathcal{C}^{\gamma}$ with $\|\phi\|:=\sup _{\theta \leq 0} e^{\gamma \theta}|\phi(\theta)|<\eta /(2 M)$, and let $u$ be any solution of (19)-(20) through $(\sigma, \phi)$. Set

$$
\{h(t)\}(x)=-(\delta / K) u(t, x)\left(\sum_{j=0}^{\infty} c(j) u([t-j], x)\right), \quad x \in[0, \pi] .
$$


Then $h$ is an $X$-valued function which is continuous except at integers, and $u$ is a (mild) solution of the abstract equation

$$
\dot{u}(t)=A u(t)+\sum_{k=0}^{\infty} B(k) u([t-k])+h(t) .
$$

Notice that $u(\cdot)=u(\cdot, \sigma, \phi ; 0)+u(\cdot, \sigma, 0 ; h)$, and hence we have

$$
u(t)=\{U(t, \sigma) \phi\}(0)+\int_{\sigma}^{t} r(t, s) h(s) d s, \quad t \geq \sigma
$$

by the preceding proposition. Observe that $|\phi(\theta)| \leq \eta e^{-\gamma \theta} /(2 M) \leq \eta e^{-\gamma \theta}$ for $\theta \in \mathbf{R}^{-}$. Then, as long as $|u(s)| \leq \eta$ on $[\sigma, t]$, we get

$$
\begin{aligned}
\sum_{j=0}^{\infty} c(j)|u([s-j])| & \leq \eta+\sum^{\prime} c(j)|\phi([s-j]-\sigma)| \\
& \leq \eta\left(1+\sum^{\prime} c(j) e^{-\gamma([s-j]-\sigma)}\right) \\
& \leq \eta\left(1+\sum_{j=0}^{\infty} c(j) e^{-\gamma(s-j-\sigma-1)}\right) \leq \eta\left(1+l e^{\gamma}\right)
\end{aligned}
$$

(where $\sum^{\prime}$ is the sum over $j$ such that $[s-j] \leq \sigma$ ), and hence

$$
|h(s)| \leq(\delta \eta / K)\left(1+l e^{\gamma}\right)|u(s)|, \quad \sigma \leq s \leq t .
$$

Therefore

$$
\begin{aligned}
|u(t)| & \leq\|U(t, \sigma) \phi\|+\left|\int_{\sigma}^{t} r(t, s) h(s) d s\right| \\
& \leq M e^{-\alpha(t-\sigma)}\|\phi\|+(\delta \eta M / K)\left(1+l e^{\gamma}\right) \int_{\sigma}^{t} e^{-\alpha(t-s)}|u(s)| d s,
\end{aligned}
$$

and applying Gronwall's Lemma we get

$$
|u(t)| \leq M\|\phi\| e^{-\left\{\alpha-\left(\delta \eta M\left(1+l e^{\gamma}\right) / K\right)\right\}(t-\sigma)}=M\|\phi\| e^{-(\alpha / 2)(t-\sigma)}<\eta
$$

as long as $|u(s)| \leq \eta$ on $[\sigma, t]$. Thus we see that $u$ satisfies $|u(t)|<\eta$ for all $t \geq \sigma$, and

$$
|u(t)| \leq M\|\phi\| e^{-(\alpha / 2)(t-\sigma)}, \quad t \geq \sigma
$$

whenever $\|\phi\|<K \alpha /\left(4 M^{2} \delta\left(1+l e^{\gamma}\right)\right)$; which shows the desired stability property.

ACKnowledgement. The authors thank the referee for his/her suggestion which enabled us to improve the proof of Proposition 4. In fact, the proof of the 
proposition in the original manuscript was rather lengthy and cumbersome. The idea for the above short proof of Proposition 4 is due to the referee.

\title{
References
}

[1] L.A.V. Carvalho and K.L. Cooke, A nonlinear equation with piecewise continuous argument, Differential and Integral Equations, 1 (1988), 359-367.

[2] K.L. Cooke and J. Wiener, Retarded differential equations with piecewise constant delays, J. Math. Anal. Appl., 99 (1984), 265-297.

[3] S. Elaydi, An Intoroduction to Difference Equations, Springer-Verlag, New York, 1996.

[4] S. Elaydi and S. Murakami, Asymptotic stability versus exponential stability in linear Volterra difference equations of convolution type, J. Difference Equations and Appl., 2 (1996), 401-410.

[5] K. Gopalsamy, Stability and Oscillations in Delay Differential Equations of Population Dynamics, Mathematics and its Applications, Kluwer Academic Publishers, 1992.

[6] S. Mohamad and K. Gopalsamy, Extreme stability and almost periodicity in a discrete logistic equation, Tôhoku Math. J., 52 (2000), 107-125; Correction, Tôhoku Math. J., 53 (2001), 629-631.

[7] I. Györi and G. Ladas, Oscillation Theory of Delay Differential Equations with Applications, Oxford Math. Monographs, Clarendon Press, Oxford, 1991.

[ 8 ] A. Pazy, Semigroups of Linear Operators and Applications to Partial Differential Equations, Applied Math. Sci. 44, Spriger-Verlag, Berlin-New York, 1983.

[9] W. Rudin, Functional Analysis, McGraw-Hill, New Delhi, 1988.

[10] J. Wiener, Generalized Solutions of Functional Differential Equations, World Scientific, Singapore, 1993.

\author{
Tetsuo Furumochi \\ Department of Mathematics \\ SHIMANE UNIVERSITY \\ MATSUE 690-8504, JAPAN \\ E-mail: furumochi@math.shimane-u.ac.jp \\ Satoru Murakami \\ Department of Applied Mathematics \\ Okayama University of Science \\ 1-1 Ridaicho, OKayama 700-0005, Japan \\ E-mail: murakami@youhei.xmath.ous.ac.jp \\ Yutaka NagabUCHI \\ Department of Systems and Control Engineering \\ Anan National College of Technology \\ ANan ToKushima 774-0017, JAPAN \\ E-mail: nagabuci@anan-nct.ac.jp \\ Present address: \\ Department of Applied Science \\ Okayama University of Science \\ 1-1 Ridaicho, OKaYama 700-0005, JaPan \\ E-mail: nagabuti@mdas.ous.ac.jp
}

